\title{
Role of steroids in modulating levels of cytokines in patients of dengue fever and warning signs
}

\begin{abstract}
Aim of Study: To define the role of corticosteroids on the levels of cytokines and immuno pathological basis for their use in dengue fever.

Materials And Methods: An open label, investigator initiated, randomized interventiona study was conducted that included 29 dengue patients (all positive for either NS1 antigen or IgM ELISA). Patients were randomised to receive a single dose of $1 \mathrm{gm}$ methyl prednisolone or $100 \mathrm{ml}$ normal saline alone. Serum cytokine levels (IL-6, TNF- $\alpha$ and sTNFR1) were measured on days 0,3 and 7 of presentation.

Results: All the three cytokines showed decline in values over the 7 day in both test as well as control groups ( $\mathrm{p}-$ value of $<0.001$ ). IL-6 and TNF- $\alpha$ levels decreased more precipitously in test group as compared to control group though the difference didn't reach statistical significance.
\end{abstract}

Conclusion: The single dose of methyl prednisolone didn't have any significant effect on the cytokine levels, but it did cause more rapid decline in levels of IL -6 and TNF- $\alpha$
Volume 5 Issue 5 - 2017

\section{Pannu AK, Bhalla A}

Department of Internal Medicine, Post Graduate Institute of Medical Education and Research, India

Correspondence: Ashish Bhalla, Professor (Internal medicine), Department of Internal medicine, 4th Floor, F Block, Post Graduate Institute of Medical Education and Research, Chandigarh, India,Tel 9| 99|4209536,9l 94I7023973; Email bhallachd@gmail.com

Received: January 28, 2017 | Published: June 15, 2017

\section{Introduction}

Dengue fever (DF), also known as break-bone fever, is a tropical infectious disease caused by the dengue virus. Dengue virus is a member of Flaviviridae family in the genus Flavivirus. ${ }^{1,2}$ The dengue virus complex comprises of four antigenic ally related viruses designated dengue virus serotypes 1 through 4 . Although DF is a self-limited febrile illness, it can progress to dengue hemorrhagic fever (DHF) in a number of patients. DHF is characterized by thrombocytopenia and increased vascular permeability leading to prominent hemorrhagic manifestations and a increased mortality. ${ }^{3}$ Analysis of serum from patients infected with dengue virus indicates that concentrations of IL-10, ${ }^{4} \mathrm{TNF}-\alpha,{ }^{5} \mathrm{IL}-8,{ }^{6} \mathrm{IL}-12,{ }^{7} \mathrm{IFN}-\Upsilon,{ }^{8} \mathrm{IFN}-\alpha .{ }^{9}$ And soluble TNF and IL -2 receptors are increased during DF and DHF. Cell cultures infected with the dengue virus release increased concentrations of inflammatory cytokines and other mediators. Prior studies have also correlated increased levels of several cytokines with disease severity and may have prognostic value. ${ }^{10-15}$ In addition, these studies show that levels of cytokines adversely affecting the coagulation cascade tend to be higher in DHF versus DF. ${ }^{10,16}$ Given the critical role of cytokines in the inflammatory process and Coagulopathy, there have been numerous attempts to suppress their levels in an attempt to control various diseases. ${ }^{17-19}$ Glucocorticoids have an inhibitory effect on a broad range of immune responses mediated by $\mathrm{T}$ cells and $\mathrm{B}$ cells, as well as potent suppressive effect on the effector functions of phagocytes. They inhibit the synthesis of almost all known cytokines (IL 1, 2, 3, 4, 5, 6, 8, 10, 13, GM-CSF, TNF- $\alpha$ and IFN-Y). Since inflammatory cytokines have been proposed to play an important role in pathogenesis of dengue fever and its various complications there has been considerable interest in studying the potential role of corticosteroids as a potential therapy for DF and DHF. We conducted a study to see the effect of corticosteroids on the levels of cytokines in dengue patients and hence provide an immuno pathological basis for the use of corticosteroids in DF, a highly debated practice with many studies giving conflicting results.

\section{Materials and methods}

The study was conducted in the medical emergency of a tertiary care hospital in northern India from July 2012 - November 2012. Patients (more than 12 years of age) with a presumptive diagnosis of DF based on fever for more than 2 days, accompanied by two or more of the following: retro-orbital pain, myalgia, arthralgia, rash, leucopenia and thrombocytopenia. ${ }^{1}$ Serum was collected and dengue infection was confirmed by detection of either NS1 antigen or dengue IgM antibodies ( $\mu$ capture) and only antigenic ally or serologically proven patients were taken up for analysis. Testing for DF was part of standard clinical protocol in our hospital given the epidemiology of the disease and the clinical presentation. A total of 29 patients who tested positive for DF and provided-informed consent were included in the study. Of the 29 patients, 17 were randomly allocated into the test group and 12 into the control group. The patients in the test group received a single dose of $1 \mathrm{gm}$ methyl prednisolone in 100 $\mathrm{ml}$ normal saline, while the control group received placebo, $100 \mathrm{ml}$ normal saline on the day of randomization. Both the patient and the drug administrator were blinded with regards to the treatment being administered. Serum cytokine levels (IL-6, TNF- $\alpha$ and sTNFR1) were measured on days 0,3 and 7 of presentation via commercially available ELISA kits and their levels were compared amongst two groups using random measures ANOVA.

\section{Results}

Baseline characteristics were measured for the two groups. Mean age of group 1 (cases) was $27.94 \pm 7.215$, while the mean age of group 2 (controls) was $35.33 \pm 10.633$, difference being non significant ( $p$ value- 0.064$)$. Sex ratio between the two groups was also comparable ( $p$ value- 0.895 ) with $82.35 \%$ males in the case group (14/17) and $83.33 \%$ males in the control group (10/12). Serum concentrations of IL- -6, TNF $-\alpha$ and TNFsR 1 were measured on day 0 , day 3 and day 7 of presentation and intra-group and intra-group analysis was performed. There was no significant difference at baseline in the test and control group for IL-6 ( $p$ value- 0.093), TNF- $\alpha$ ( $p$ value- 0.797$)$ and TNFsR1 ( $p$ value- 0.587 ). All the three cytokines showed significant decline in values over the seven day study period with a $\mathrm{p}-$ value of $<0.001$ in both test as well as control groups (Table 1) and (Figures $1-3)$. Looking at the percentage decline over the first three days, it was found that IL -6 and TNF- $\alpha$ levels decreased more precipitously in test group as compared to control group as can be accessed from the slopes of figures 1and 2, though the difference didn't reach statistical significance (Table 2). The single dose of methyl prednisolone didn't 
have any statistically significant effect on the cytokine levels, but it did cause more rapid decline in levels of IL -6 and TNF- $\alpha$.

Table I Mean level of cytokines (values in $\mathrm{pg} / \mathrm{ml}$ )

\begin{tabular}{|c|c|c|c|c|c|c|c|c|}
\hline \multirow[t]{2}{*}{ Group } & \multicolumn{4}{|c|}{ IL-6 } & \multicolumn{2}{|l|}{ TNF- $\alpha$} & \multicolumn{2}{|c|}{ TNFsR I } \\
\hline & Day 0 & \multicolumn{3}{|c|}{$149.520 \pm 120.19$} & \multicolumn{2}{|c|}{$473.088 \pm 220.91$} & \multicolumn{2}{|c|}{$935.56 \pm 114.44$} \\
\hline \multirow[t]{3}{*}{ Test } & Day 3 & \multicolumn{3}{|c|}{$73.539 \pm 74.63$} & \multicolumn{2}{|c|}{$293.088 \pm 107.25$} & \multicolumn{2}{|c|}{$883.81 \pm 154.48$} \\
\hline & Day 7 & \multicolumn{3}{|c|}{$32.755 \pm 42.02$} & \multicolumn{2}{|c|}{$215.441 \pm 84.02$} & \multicolumn{2}{|c|}{$821.94 \pm 197.85$} \\
\hline & Day 0 & \multicolumn{3}{|c|}{|| $5.36| \pm 34.0|$} & \multicolumn{2}{|c|}{$422.083 \pm 231.27$} & \multicolumn{2}{|c|}{$930.51 \pm 126.85$} \\
\hline \multirow[t]{2}{*}{ Control } & Day 3 & \multicolumn{3}{|c|}{$64.111 \pm 36.13$} & \multicolumn{2}{|c|}{$299.167 \pm 101.38$} & \multicolumn{2}{|c|}{$853.15 \pm 203.68$} \\
\hline & Day 7 & \multicolumn{3}{|c|}{$30.639 \pm 27.42$} & \multicolumn{2}{|c|}{$229.583 \pm 46.10$} & \multicolumn{2}{|c|}{$745.86 \pm 210.33$} \\
\hline \multicolumn{3}{|c|}{ Study Group } & $\mathbf{N}$ & \multicolumn{2}{|c|}{ Mean (\% age) } & \multicolumn{2}{|c|}{ Std. Deviation } & $P$ value \\
\hline \multirow{2}{*}{ IL-6 } & \multicolumn{2}{|c|}{ Control Group } & 12 & \multicolumn{2}{|c|}{45.5508} & \multicolumn{2}{|c|}{20.9132} & \multirow{2}{*}{0.696} \\
\hline & Test Gro & & 17 & 49.09 & & 27.376 & & \\
\hline \multirow{2}{*}{ TNF- $\alpha$} & \multirow{2}{*}{\multicolumn{2}{|c|}{$\begin{array}{l}\text { Control Group } \\
\text { Test Group }\end{array}$}} & 12 & \multicolumn{2}{|c|}{22.6407} & \multicolumn{2}{|c|}{21.2121} & \multirow{2}{*}{0.228} \\
\hline & & & 17 & 32.27 & 764 & 19.774 & & \\
\hline \multirow{2}{*}{ TNFsR I } & Control & Group & 12 & 9.47 & & 15.372 & & \\
\hline & Test Gro & & 17 & 5.636 & & 12.155 & & 0.40 \\
\hline
\end{tabular}

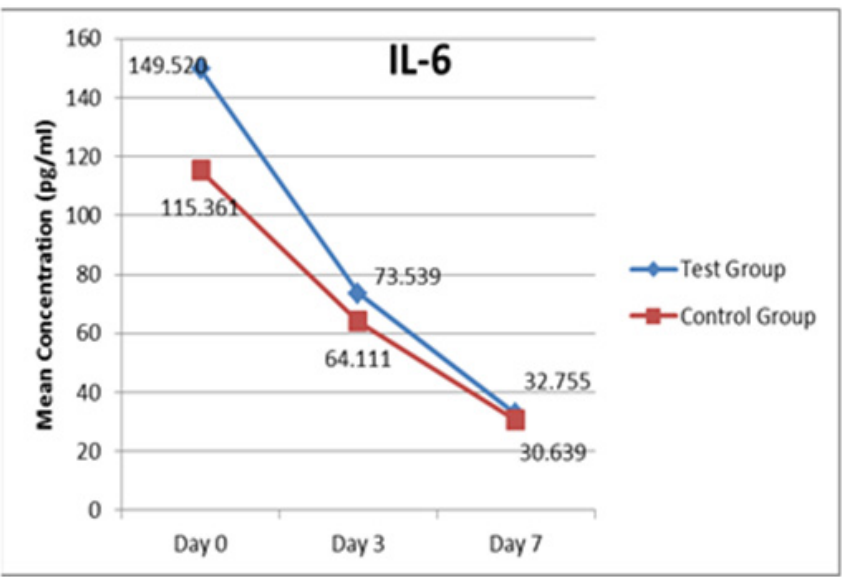

Figure I IL-6 Levels: Levels decrease over 7 day study period in both the study groups with levels being maximum at presentation and minimum at day 7. This intra-group difference is statistically significant with $p-v a l u e ~<0.00$ I.

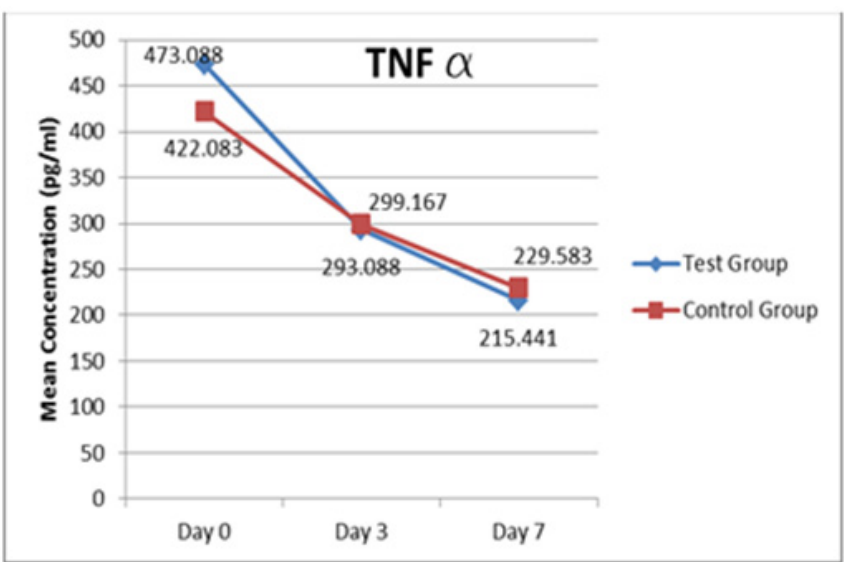

Figure 2 TNF $\alpha$ Levels: Levels decrease over 7 day study period in both the study groups with levels being maximum at presentation and minimum at day 7. This intra-group difference is statistically significant with $p-v a l u e ~<0.00$ I.

\section{Discussion}

Deregulation of the immune system has been well established in the pathogenesis of DF and hence corticosteroids are often used for treatment of severe cases of DF or DHF. Although there is no data about their efficacy from prospective studies but there are several case reports that recount anecdotal experiences of clinical benefit derived from steroids in severe cases of DF. Recently Premaratna et al. ${ }^{20}$ described a case of a 14-year-old boy with dengue who developed three episodes of severe hemodynamic compromise while having high fever, myocarditis, third space fluid accumulation, progressive reduction in urine output, and altered mutation, who made a dramatic recovery following a single dose of intravenous methyl prednisolone. ${ }^{20}$ With this study, we aimed to provide an immuno pathological basis for the use of corticosteroids in DF.

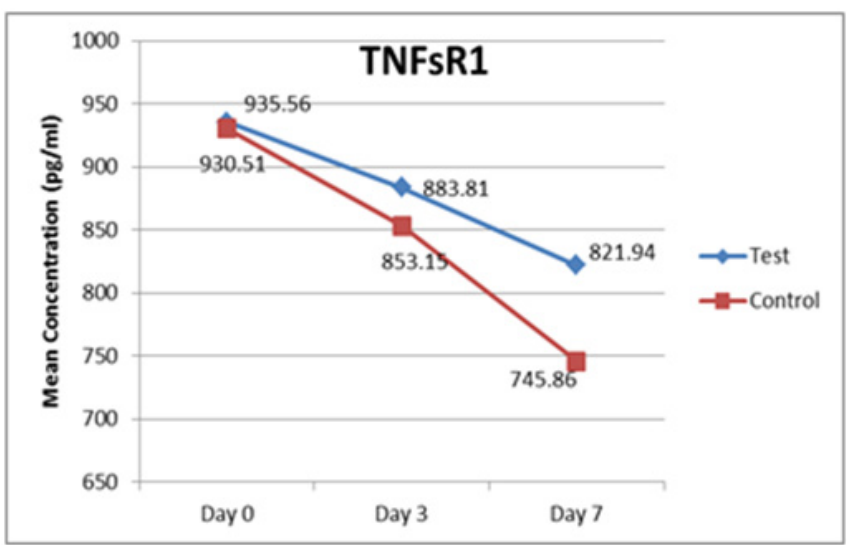

Figure 3 TNFsR I Levels: Levels decrease over 7 day study period in both the study groups with levels being maximum at presentation and minimum at day 7.This intra-group difference is statistically significant with $\mathrm{p}-$-value $<0.00$ I.

Numerous cytokines have been shown to play an important role in the pathogenesis ofDF. We studied the levels of three different cytokines shown in different studies to be increased in DF patients as compared to healthy controls, namely IL $-6, \mathrm{TNF}-\alpha$ and soluble TNF Receptor 1 (TNFsR1). In a study to understand the pathogenesis of the disease, an in vitro model utilizing peripheral blood mononuclear leukocytes were infected with the dengue virus and levels of cytokines studied. The study showed increased levels of TNF- $\alpha$, IL -6, IL- -10 and IFN- $\alpha$ in the supernatant of infected cells. Furthermore, treatment of cell cultures with dexamethasone led to a significant decrease in levels of TNF $-\alpha$, IL -10 and IFN $-\alpha$ when compared to untreated cultures. IL -6 levels in supernatants from dexamethasone-treated cell cultures were not statistically different from infected control cultures. ${ }^{21}$ In contrast, a recently published study showed that the levels of 11 cytokines and chemokine did not show a difference in levels between 3 treatment groups- receiving high-dose corticosteroids (defined as $2 \mathrm{mg} / \mathrm{kg}$ oral prednisolone), low-dose corticosteroids (defined as $0.5 \mathrm{mg} / \mathrm{kg}$ oral prednisolone) or identical placebo- 2 days after starting therapy. ${ }^{22}$ Our study showed that levels of all the three cytokines progressively decreased over the period of 7 days, as the dengue patients recovered, implying an increased production of these cytokines early in the course of the disease. Role of corticosteroids in reducing the levels of these cytokines was evaluated and we found that levels of all the three cytokines were not significantly different amongst the two groups the levels at all time points. Further evaluation revealed that the fall in the levels of IL- 6 and TNF- $\alpha$ over first three days was more precipitous in test-group patients as compared to control patients, but the difference didn't reach statistical significance. Our results are important for several reasons. The use of corticosteroids for potentially reverses the pro-inflammatory state in DF and/or DHF is highly debated and there is no strong clinical evidence either way. We show that use of high-dose prednisolone did not appreciably impact the rate of change in three pro-inflammatory cytokines that 
have been previously implicated in pathogenesis of DF. Our findings could explain the lack of clinical benefit with steroids in this disease setting. However we collected only 29 patients in this study and other cytokines which have also been implicated in DF pathogenesis were not evaluated. Further exploration of the impact of steroids on the clinical and immunologic parameters in DF is required.

\section{Acknowledgments}

None.

\section{Conflicts of interest}

None.

\section{References}

1. Henchal EA, Putnak JR The dengue viruses. Clin Microbiol Rev. 1990;3(4):376-396

2. Wilder-Smith A, Schwartz E Dengue in travelers. $N$ Engl J Med. 2005;353:924-932.

3. Deen JL, Harris E, Wills B et al. The WHO dengue classification and case definitions: time for a reassessment. Lancet. 2006;368(9530):170-173.

4. Green S, Vaughn DW, Kalayanarooj S et al. Elevated plasma interleukin-10 levels in acute dengue correlate with disease severity. $J$ Med Virol. 1999;59:329-334.

5. Hober D, Nguyen TL, Shen L et al. Tumor necrosis factor alpha levels in plasma and whole-blood culture in dengue-infected patients: relationship between virus detection and pre-existing specific antibodies. J Med Virol. 1998;54(3):210-218.

6. Raghupathy R, Chaturvedi UC, Al-Sayer H et al. Elevated levels of IL-8 in dengue haemorrhagic fever. J Med Virol. 1998;56(3):280-285.

7. Pacsa AS, Agarwal R, Elbishbishi EA et al. Role of interleukin-12 in patients with dengue hemorrhagic fever. FEMS Immunol Med Microbiol. 2000;28(2):151-155.

8. Libraty DH, Endy TP, Houng HS et al. Differing influences of virus burden and immune activation on disease severity in secondary dengue-3 virus infections. J Infect Dis. 2002;185:1213-1221.

9. Kurane I, Innis BL, Nimmannitya S et al. High levels of interferon alpha in the sera of children with dengue virus infection. Am J Trop Med Hyg 1993;48(2):222-229.
10. Green S, Vaughn DW, Kalayanarooj S et al. Early immune activation in acute dengue illness is related to development of plasma leakage and disease severity. J Infect Dis. 1999;179(4):755-762.

11. Marty AM, Jahrling PB, Geisbert TW viral hemorrhagic fevers. Clin Lab Med. 2006;26(2):345-386.

12. Atrasheuskaya AV, Fredeking TM, Ignatyev GM Changes in immune parameters and their correction in human cases of tick-borne encephalitis. Clin Exp Immunol. 2003;131(1):148-154.

13. Bozza FA, Cruz OG, Zagne SM et al. Multiplex cytokine profile from dengue patients: MIP-1beta and IFN-gamma as predictive factors for severity. BMC Infect Dis. 2008;8:86.

14. Bethell DB, Flobbe K, Cao XT et al. Pathophysiologic and prognostic role of cytokines in dengue haemorrhagic fever. $J$ Infect Dis. 1998;177(3):778-782.

15. Hober D, Poli L, Roblin B et al. Serum levels of tumor necrosis factor- $\alpha$ $(\mathrm{TNF}-\alpha)$, interleukin-6 (IL-6), and interleukin-1 $\beta$ (IL-1 $\beta$ ) in dengueinfected patients. Am J Trop Med Hyg. 1993;48(3):324-331.

16. Suharti C, van Gorp EC, Setiati TE et al. The role of cytokines in activation of coagulation and fibrinolysis in dengue shock syndrome. Thromb Haemost. 2002;87(1):42-46.

17. Feldmann M Many cytokines are very useful therapeutic targets in disease. J Clin Invest. 2008;118(11):3533-3536.

18. Phelan JD, Orekov T, Finkelman FD. Cutting edge: mechanism of enhancement of in vivo cytokine effects by anticytokine monoclonal antibodies. J Immunol. 2008;180(1):44-48.

19. Ratsimandresy RA, Rappaport J, ZaguryJF. Anticytokine therapeutics: history and update. Curr Pharm Des. 2009;15(17):1998-2025.

20. Premaratna R, Rodrigo KM, Anuratha A, et al. Repeated dengue shock syndrome and 'dengue myocarditis' responding dramatically to a single dose of methyl prednisolone. Int J Infect Dis. 2012;16(7):e565-569.

21. Reis RN, Sampaio AL, Henriques Md, et al. An in vitro model for dengue virus infection that exhibits human monocyte infection, multiple cytokine production and dexamethasone immunomodulation Mem Inst Oswaldo Cruz, Rio de Janeiro, December. 2007;102(8):983-990.

22. Nguyen TH, Nguyen TH, Vu TT, et al. Corticosteroids for Dengue Why Don't They Work? PLoS Negl Trop Dis. 2013;7(12):e2592. 